\title{
Performance of the ATLAS Calorimeters in LHC Run-1 and Run-2
}

\author{
Blake Burghgrave* \\ Northern Illinois University \\ E-mail: blake.burghgravedcern.ch
}

\section{On behalf of the ATLAS Collaboration}

The ATLAS experiment at the Large Hadron Collider (LHC) is equipped with electromagnetic and hadronic liquid-argon (LAr) calorimeters and a hadronic scintillator-steel sampling calorimeter (TileCal) for measuring energy and direction of final state particles in the pseudorapidity range $|\eta|<4$.9. The calibration and performance of the calorimetry system was established through beam tests, cosmic ray muon measurements and in particular the first three years of pp collision data-taking. During this period, referred to as Run-1, approximately $27 \mathrm{fb}^{-1}$ of proton-proton collision data were collected at the center-of-mass energies of 7 and $8 \mathrm{TeV}$. Following a period of detector consolidation during a long shutdown, Run-2 started in 2015 with approximately $3.9 \mathrm{fb}^{-1}$ of data at a center-of-mass energy of $13 \mathrm{TeV}$ recorded in the first year. We present a summary of the calorimeter operation, monitoring and data quality, as well as their performance, including the calibration and stability of the electromagnetic scale and time resolution. These results demonstrate that the LAr and Tile calorimeters perform excellently within their design requirements. The calorimetry system thus played a crucial role in the Run-1 physics programme, and, in particular, in the discovery of a Higgs boson.

38th International Conference on High Energy Physics

3-10 August 2016

Chicago, USA

${ }^{*}$ Speaker. 


\section{Introduction}

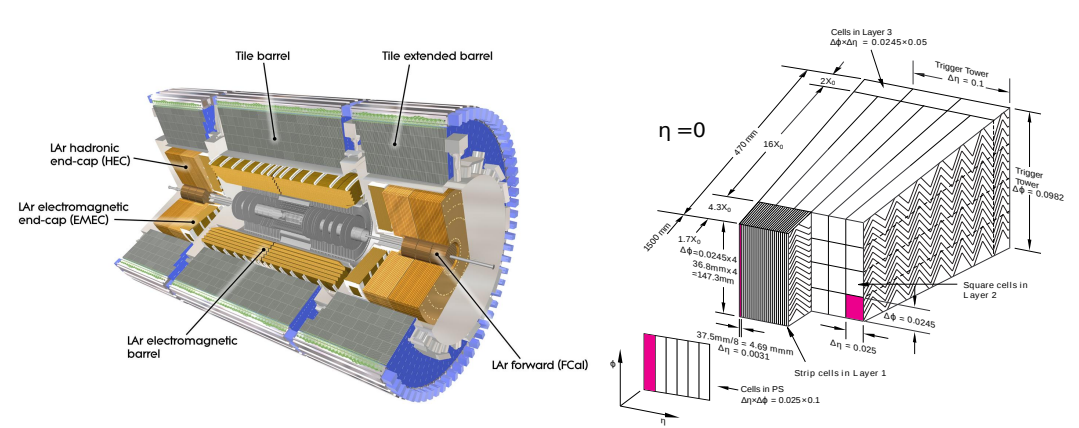

(a) ATLAS Calorimeters

(b) $\mathrm{LAr}$

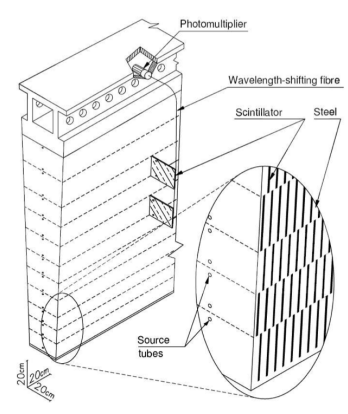

(c) Tile

Figure 1: The ATLAS calorimeter systems (a) [2] consist of the Liquid Argon Calorimeter (b) [3] and the Tile Calorimeter (c) [4].

\subsection{Liquid Argon Calorimeter}

The ATLAS [1] Liquid Argon (LAr) calorimeter is a sampling calorimeter segmented into up to 4 layers by depth, with Liquid Argon as active material, and either lead, copper, or a tungsten/copper alloy as passive material in the electromagnetic barrel (EMB) and endcap (EMEC), the hadronic endcap (HEC), and forward calorimeter (FCal) regions, respectively. Liquid Argon is ionized by high-energy charged particles. The freed electrons drift to copper readout electrodes. The LAr EMB covers $|\eta|<1.5$, the EMEC+HEC cover $1.4<|\eta|<3.2$, and the FCal covers the $3.1<|\eta|<4.9$ region. In total, LAr has approximately 180,000 readout channels.

\subsection{Tile Calorimeter}

The Tile Calorimeter envelopes the LAr calorimeter the $|\eta|<1.7$ region, including cells in the crack region between the Tile long barrel and extended barrel, and the LAr EMB and HEC sections. Scintillator tiles and steel absorbers are used to measure the energy of (primarily hadronic) particles, and are read out with approximately 10,000 photo-multiplier tubes (PMTs). Minimum Bias Trigger Scintillator (MBTS), used by the trigger system in low-luminosity runs, covers the 2.1 $<|\eta|<3.9$ region.

\section{Calibration and Noise}

LAr calibration was established with high precision and stability in Run-1, with the electronic baseline (pedestal) stable to within $0.02-0.03$ ADC counts and relative gains in the $0.05-0.20$ per mill range [5]. LAr noise is well understood and modeled accurately in Monte Carlo simulation (Fig. 2a).

A Cs-137 radioactive source is used to calibrate Tile gains. The Laser calibration system [6] is used to correct for PMT drifts between Cs scans, validated with gains seen in the Minimum Bias integrator system. Laser, Charge Injection, and Pedestal calibration runs are used to monitor timing, stability, and noise (Fig. 2c) [7]. Beam splash events [7], collected before the start of data 


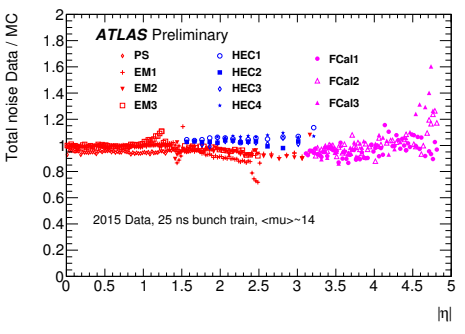

(a) LAr noise

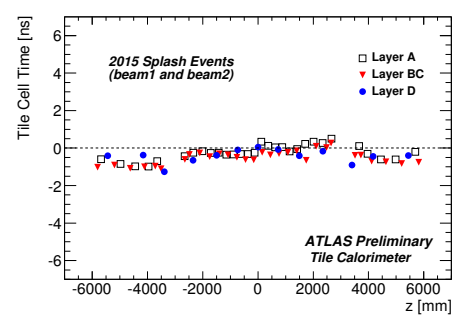

(b) Tile timing

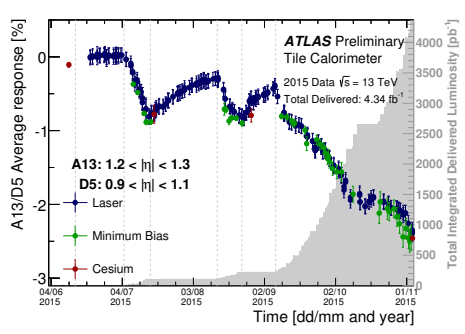

(c) Tile response

Figure 2: (a) Ratio of total noise in LAr (electronics and pile-up for $\langle\mu\rangle=14$ ) at the electron scale (data/MC) [3]. (b) Timing of Tile Calorimeter signals recorded with single beam data on April 2015 [4]. (c) The variation of the response to Minimum Bias, Cesium and Laser for cells in the inner layer of the Extended Barrel, covering the region $1.2<|\eta|<1.3$, as a function of time [4].

taking, are used to uniform the timing response of the Tile Calorimeter (Fig. 2b). Changes to Tile timing during collision runs are monitored by using Laser calibration events in the empty bunch crossings of Physics runs, and are used to apply timing offset corrections to data.

\section{Monitoring}

Impurities in LAr can lead to electron attachment, causing a reduction of measured signal, and must remain below $1 \mathrm{ppm}$. Purity monitors track the long term stability of LAr impurities every 10-15 minutes when outside data taking (Fig. 3a).

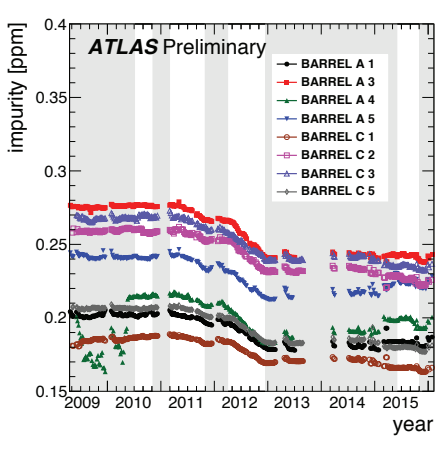

(a) LAr impurities

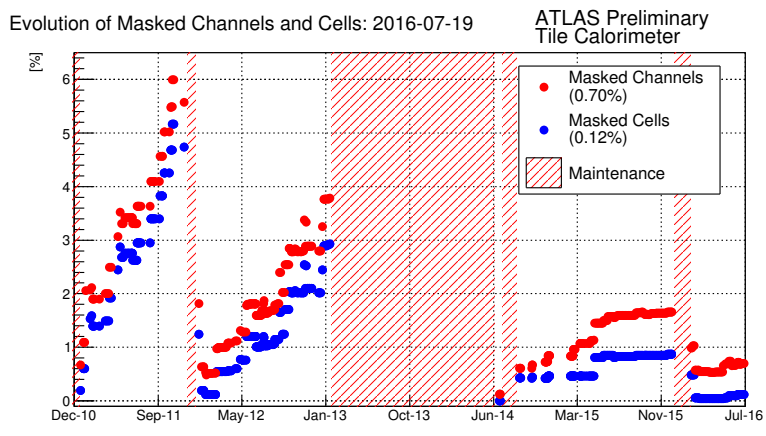

(b) Tile masked channels and cells

Figure 3: (a) Measured impurity of the liquid argon in the barrel cryostat [3]. (b) The percentage of all cells and channels in the Tile Calorimeter that are masked [4].

Coherent "noise bursts" in LAr appear as temporary correlated noise in a large fraction of an EMB or EMEC partition, which causes a brief loss of coverage in the calorimeter, making the data collected at that time unsuitable for use in physics analyses. These bursts are identified in cosmic data taking, and used to veto the affected time window in collision data. Automatic flagging of noise bursts reduced the affected data from $1.46 \%$ in 2011 to $0.26 \%$ in 2012 and $0.05 \%$ in 2015 . A new class of "mini-noise-bursts", affecting a smaller region of the calorimeter, emerged in 2015 as the second largest contributor to LAr data loss in that year. Automatic flagging has improved the situation in 2016. 
A fraction of Tile data losses in Run-1 were related to Low-Voltage Power Supply (LVPS) trips. Redesigned LVPS units were tried on a part of the detector in 2012, and found to virtually eliminate the problem. All older units were then replaced by the new ones during the LHC Long Shutdown 1 maintenance and upgrade period. This exercise is also thought to have led to a significant reduction in the number of bad channels from Run-1 to Run 2 (Fig. 3b). No Tile data losses were incurred in 2015. A $25 \mathrm{~ns}$ timing jump in 1/4 of Tile is the most significant issue to occur so far in 2016. Tile monitoring includes identifying and masking problematic channels, correcting for timing jumps, monitoring hardware issues, and (in Run-2) monitoring and correcting for changes in pedestal.

\section{Performance}

Time resolution for the LAr EM sections are found to have an approximately 200 ps correlated contribution from the beamspot width, in addition to uncorrelated contributions of about $170 \mathrm{ps}$ for the EMB and about 65 ps for the EMEC [3]. Time resolution in Tile is calculated using reconstructed jets with $p_{\mathrm{T}}>20 \mathrm{GeV}$, with slightly better timing in the Long Barrel region (Fig. 4a) than the Extended Barrel (due to smaller cell sizes). Beam splash events are used to test detector timing and response uniformity (Fig. 4b).

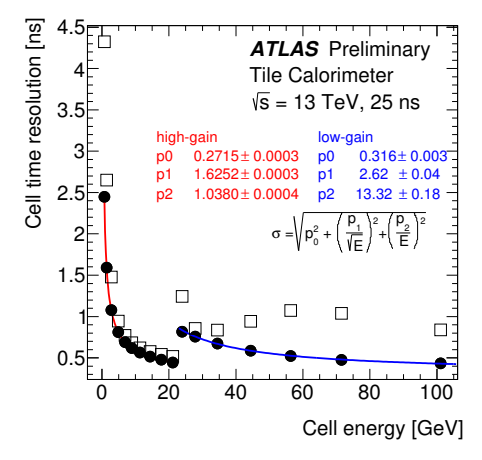

(a) Tile time resolution

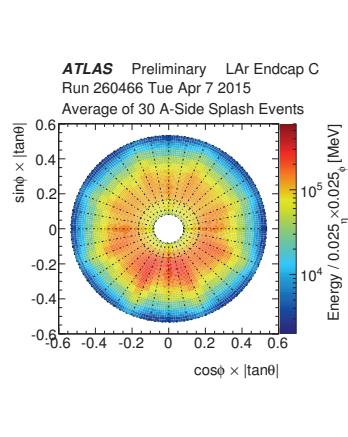

(b) LAr energy response

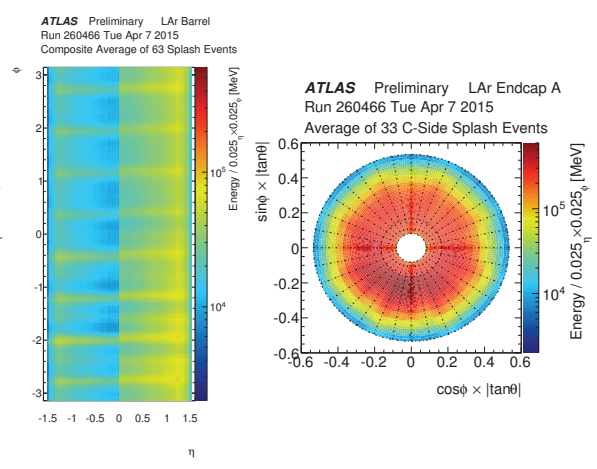

Figure 4: (a) Tile cell time resolution in jet events [4]. (b) LAr energy response uniformity in beam splash events [3], with an eight-fold banding pattern corresponding to shadows from the toroid magnets.

\section{References}

[1] ATLAS Collaboration, 2008 JINST 3 S08003

[2] ATLAS Collaboration, http://atlasexperiment.org/photos/calorimeters-combined-barrel.html

[3] ATLAS Collaboration, https://twiki.cern.ch/twiki/bin/view/AtlasPublic/ApprovedPlotsLAr

[4] ATLAS Collaboration, https://twiki.cern.ch/twiki/bin/view/AtlasPublic/ApprovedPlotsTile

[5] H. Abreu et al., 2010 JINST 5 P09003

[6] ATLAS Collaboration, 2016 JINST 11 T10005

[7] ATLAS Collaboration, Eur. Phys. J. C (2010) 70: 1193. doi:10.1140/epjc/s10052-010-1508-y 